\title{
TWO-PHASE BUBBLE FLOW AND CONVECTIVE MASS TRANSFER IN WATER SPLITTING PROCESSES
}

\author{
O. A. Jianu ${ }^{1 *}$ M. A. Rosen ${ }^{1}$, G. F. Naterer ${ }^{2}$, Z. Wang ${ }^{1}$ \\ Ofelia.Jianu@uoit.ca, Marc.Rosen@uoit.ca,gnaterer@mun.ca, Forest.Wang@uoit.ca
}

\begin{abstract}
When hydrogen or oxygen is produced from water splitting by electrolysis, thermochemical cycles or solar-based photocatalytic methods, bubble flow and vapour transfer into the gas phase occur during phase transition. This undesirable vapour transfer requires the use of more energy input to compensate for the evaporation heat requirement as well as for subsequent gas purification in the downstream unit. In this paper, both experimental and modeling studies are performed to examine the dynamics of bubble flows and kinetics of water vapour transfer, particularly related to processes of hydrogen production. Experimental data are obtained using an advanced laser-based shadow imaging system and on-line vapour monitoring system. The bubble dynamics and water vapour transfer kinetics are modeled with non-dimensional parameters involving the bubble diameter, velocity and trajectories so that the water vapour transfer rate can be quantified under different operating conditions for various hydrogen production methods. Also, a predictive model is developed to simulate the physical processes of bubble transport in a vertical liquid column, as it occurs in water splitting processes such as oxygen generation in the thermochemical copper-chlorine cycle, as well as hydrogen generation in electrolytic and photocatalytic processes.
\end{abstract}

\section{INTRODUCTION}

Many environmental problems are related to the production, transformation and use of energy. Some of the concerns include, but are not limited to, acid rain, ozone depletion and climate change. Therefore, "greener” alternatives for energy production are sought. Hydrogen energy systems are potentially effective solutions and can have a significant role in providing better environmental sustainability [1]. However, current methods of hydrogen production are often unsustainable as they are based primarily on fossil fuels such as natural gas or coal, which release $\mathrm{CO}_{2}$ into the atmosphere.

\footnotetext{
${ }^{1}$ Faculty of Engineering and Applied Science, University of Ontario Institute of Technology 2000 Simcoe Street North, Oshawa, Ontario, Canada

${ }^{2}$ Faculty of Engineering and Applied Science, Memorial University of Newfoundland, St. John's, Newfoundland, A1B $3 X 5$, Canada
} 
Promising alternatives for sustainable hydrogen production are thermochemical, electrolytic, and photocatalytic cycles.

Thermochemical cycles for hydrogen production use a series of reactions to achieve the overall splitting of water into hydrogen and oxygen:

$$
\mathrm{H}_{2} \mathrm{O} \rightarrow \mathrm{H}_{2}+\frac{1}{2} \mathrm{O}_{2}
$$

Thermochemical water decomposition generally involves at least three distinct steps: hydrogen production, oxygen production, and recycling of auxiliary compounds. One of the advantages of thermochemical cycles over direct one-step thermal water decomposition is their ability to achieve water splitting into hydrogen and oxygen at much lower temperatures (usually below $1,000^{\circ} \mathrm{C}$ ) [2]. One of the promising thermochemical cycles that operates at even lower temperatures is the thermochemical $\mathrm{Cu}-\mathrm{Cl}$ cycle. This cycle splits water into hydrogen and oxygen through intermediate copper and chlorine compounds. Several variations of the $\mathrm{Cu}-\mathrm{Cl}$ cycle have been reported, including 5-step, 4-step and 3-step cycles [3, 4]. The chemical reactions form a closed internal loop and recycle all chemicals on a continuous basis, without emitting any greenhouse gases externally to the atmosphere.

Another method of water splitting is conventional water electrolysis. It yields hydrogen at about 99.99\% purity compared with 98\% purity obtained from fossil fuel based methods [5]. The overall efficiency of hydrogen production through water electrolysis depends on two factors: electricity-to-hydrogen efficiency and electricity generation efficiency [6]. Past research showed that the electrical and thermal properties of the electrolyte, diffusive transport of electroactive species and current density are modified by the dispersed phase (i.e. gas bubbles). These affect the macroscopic cell performance [7]. Thus, the understanding of gas-liquid flows in electrolytic systems is important from the view-point of understanding mass transport, system optimization and improving the efficiency.

The gases released at the electrode surface rise upward due to buoyancy. The presence of the gas phase at the electrodes can be detrimental to the overall performance of the process as it blocks the active surface area of the electrodes and increases the resistance of the electrolyte [8]. The presence of gas bubbles and their motion has a significant impact on the performance of the electrolytic cell [9]. Ali and Pushpavanam [10] studied the effects of gas evolution on hydrodynamics of water electrolysis in a partitioned electrolytic system using PIV. The results were verified further quantitatively by analyzing time averaged velocity profiles along a line. The temporal variation of liquid velocity at a point was also analyzed. It was found that velocity components exhibit turbulent fluctuations about a mean value [11]. 
Gas bubbles exist in gas-liquid, gas-solid, and gas-liquid-solid systems such as the prior systems. Studies by Clift at al. [12] and Duineveld [13] reported that bubbles with diameters less than $1 \mathrm{~mm}$ rise through water and retain their spherical shape due to surface tension. Also, the trajectory follows a nearly straight line. However, this is not the case for larger bubbles. Researchers reported deformations due to the increase in hydrostatic and dynamic pressures over a bubble's surface [14], [15]. Therefore, large bubbles first deform into oblate spheroids, then become ellipsoidal, and with further increase in size, switch into a spherical or ellipsoidal cap shape [16]. The gas-phase density can be increased by increasing the pressure or using a higher molecular weight gas. As a result of increased gas density, the difference between the densities of two phases decreases, resulting in smaller sized bubbles and also a reduction in the buoyancy force [17]. According to this model, the bubble volume at the end of detachment remains the same for all bubbles generated from all holes on a porous plate [18]. Since the sizes of bubbles after its formation and its wake affect the rise velocity and direction of rise, i.e., trajectory in the liquid, they influence the above-mentioned dynamic processes, overall turbulence in the system and performance of the equipment to some extent [17, 19, 20].

With rising bubbles, past studies have shown a rectilinear path becomes a zigzag or spiral pattern, then quasi-rectilinear as the bubble size increases until it becomes a spherical cap. This has been observed in liquids of small Morton number [16]. However, the bubble size does not affect the trajectory in large Morton numbers as a rectilinear path is observed regardless of diameter [21]. Researchers also observed rocking motions for different Reynolds numbers [22]. Ohta et al. [19] discussed the trajectory of a larger bubble consisting of primary and secondary structures. The primary structure is defined as the structure where the bubble begins to oscillate and rise in a zigzag motion as soon as the bubble is released and reaches the terminal velocity. The secondary structure follows soon after when the bubble attains a special shape at terminal velocity.

The presence of water vapour in the produced hydrogen or oxygen gas indicates a latent heat loss in the reactor and a power requirement for the separation of hydrogen and water vapour in the downstream of the reactor. This may influence the hydrogen production efficiency. So the water vapour entrainment in the bubbling processes should be studied. However, these past investigations on the interactions of bubble flow dynamics and mass transfer between the liquid and bubbles are limited in their abilities and scope of predicting vapour entrainment during water splitting processes. In this paper, vapour entrainment for ascending bubbles in a vertical column is examined experimentally for various gas production rates on the basis of past studies [23]. The dependence of vapour entrainment on the phase transition rate, which is influenced by gas bubble size, liquid depth, temperature and concentration, is investigated experimentally. The objective of this paper is to develop a phase transition correlation to relate the mass transfer rate to the parameters influencing it. 


\section{EXPERIMENTAL APPARATUS}

In order to examine the water vapour generation and flow characteristics of gas-liquid systems, an experimental apparatus has been designed and built at the University of Ontario Institute of Technology (UOIT) in the Clean Energy Research Laboratory (CERL). The experimental loop examines bubble dynamics through different liquids as well as the amount of mass transferred from the liquid to the gas that is carried out to the surface of the liquid while the bubble is rising. In an electrolytic step of the $\mathrm{Cu}-\mathrm{Cl}$ cycle, water is split to produce hydrogen by means of an electric current. Hydrogen gas is produced on the cathode of the electrolyzer, whereas oxygen gas is produced on the anode. The gas bubbles rise to the surface, carrying a certain amount of moisture from the water.

The experimental loop was designed in order to quantify the amount of moisture removed during electrolysis. The apparatus consists of a $2.708 \mathrm{~m}$ high, $203 \mathrm{~mm}$ diameter, clear PVC vertical column filled with liquid. Nitrogen gas is supplied and bubbles are generated through a porous surface. The size and number of bubbles vary with the nitrogen flow rate such that less bubbles with bigger diameters are formed for lower flow rates, while more bubbles with smaller diameters are formed for higher flow rates. The flow rate of nitrogen is set manually using rotameters. Experimental data was recorded for four different flow rates to develop the correlation (i.e. $0.5 \mathrm{~L} / \mathrm{min}, 1 \mathrm{~L} / \mathrm{min}, 1.5$ $\mathrm{L} / \mathrm{min}$ and $2 \mathrm{~L} / \mathrm{min}$ ) and two flow rates to test the correlation (i.e. $0.25 \mathrm{~L} / \mathrm{min}$ and $3 \mathrm{~L} / \mathrm{min}$ ). Bubbles flowing through the porous surface rise up through the water. Due to the motion of a bubble through water, some of the water molecules from the liquid diffuse across the boundary of the bubble and they are carried out of the water column. In order to determine the amount of water carried to the surface, a humidity analyzer was installed at the gas exit. Type $\mathrm{T}$ thermocouples have also been installed at five different locations in order to record the temperature of the water as well as the temperature of the gas. LabVIEW software was used to view and record the data captured by the humidity sensor and thermocouples. A schematic of the experimental setup is presented in Figure 1.

In order to better understand the flows with bubble generation and dynamics, it is important to map the bubble size, bubble number, and bubble velocity as bubbles rise through the liquid. Hence, DynamicStudio Shadow Sizer was employed to measure the bubble dynamics. The particles rising through the medium are backlit with an LED light source with a trigger frequency of $400 \mathrm{~Hz}$, pulse of $100 \mu$ s and delay of $10 \mu \mathrm{s}$. A glass diffuser is used in order to obtain an even light sheet so that the camera can acquire a shadow image of the moving particles. A FlowSense 2ME camera with an effective sensor size of 1600 by 300 pixels was used to capture images. Images were captured in single frame mode with a trigger frequency of $91 \mathrm{~Hz}$, delay to open of $3.5 \mu$ s, delay to close of $10,920 \mu \mathrm{s}$ and exposure time of $40.950 \mu \mathrm{s}$. The particle in motion was frozen with the aid of a short light flash and a sychronization device. The timer box for imaging applications started automatically 
so that the camera and LED light array were synchronized at the start of the acquisition process. The camera and light system were set up as shown in Figure 1. The stands were custom built so that images can be taken at precise specified heights from the gas exit.

All bubbles from the acquired images were analyzed by the software by the choice in the grey level profile. The results obtained after analysis of the images are listed in Table 1. The setup was designed according to Refs. [24-26] so that the uncertainty associated with the experiment can be minimized. The uncertainty, $\mathrm{U}$, of the experimental results is determined by:

$$
\mathrm{U}_{\mathrm{i}}=\sqrt{\mathrm{B}_{\mathrm{i}}^{2}+\mathrm{P}_{\mathrm{i}}^{2}}
$$

where $B$ and $P$ represent the bias and precision error, respectively.

Consistent operating conditions are maintained during the uncertainty analysis, and the relative bias error is taken as the ratio of the bias error to the corresponding reference value. The limit is calculated as follows:

$$
\begin{aligned}
B_{S h}^{2} & =\left(\frac{\partial S h}{\partial D}\right)^{2} B_{e q}^{2}+\left(\frac{\partial S h}{\partial H}\right)^{2} B_{H}^{2}+\left(\frac{\partial S h}{\partial V}\right)^{2} B_{V}^{2}+ \\
& \left(\frac{\partial S h}{\partial \sigma}\right)^{2} B_{\sigma}^{2}+\left(\frac{\partial S h}{\partial \mu}\right)^{2} B_{\mu}^{2}+\left(\frac{\partial S h}{\partial g}\right)^{2} B_{g}^{2}+\left(\frac{\partial S h}{\partial \rho}\right)^{2} B_{\rho}^{2}
\end{aligned}
$$

The measurement devices used in the experiments have a relatively high accuracy and low bias error associated with their operation.

The precision error is double the standard deviation of the results [24]. A sample of 50 measurements at equilibrium conditions was considered for the precision error calculations. The propagation of precision error was determined as follows:

$$
\begin{aligned}
P_{S h}^{2} & =\left(\frac{\partial S h}{\partial D}\right)^{2} P_{e q}^{2}+\left(\frac{\partial S h}{\partial H}\right)^{2} P_{H}^{2}+\left(\frac{\partial S h}{\partial V}\right)^{2} P_{V}^{2}+ \\
& \left(\frac{\partial S h}{\partial \sigma}\right)^{2} P_{\sigma}^{2}+\left(\frac{\partial S h}{\partial \mu}\right)^{2} P_{\mu}^{2}+\left(\frac{\partial S h}{\partial g}\right)^{2} P_{g}^{2}+\left(\frac{\partial S h}{\partial \rho}\right)^{2} P_{\rho}^{2}
\end{aligned}
$$

The experimental errors due to the equipment (i.e., camera calibration, residual, resolution and PVC vessel wall distortion) and analysis, as well as the deviations, are shown in Table 2.

\section{BUBBLE FLOW AND MASS TRANSFER MODEL}

\subsection{Assumptions}

Various assumptions similar to past studies [17] were adopted. It was assumed that the wall does not influence the ascending gas bubbles due to the large distance between the bubbles and the wall. Since the volume of the liquid within the column was much larger than the volume of the bubbles formed, it was assumed that the liquid is a semi-infinite medium in comparison to bubble volume. Also, the added mass coefficient of the liquid is assumed constant and since the Galileo number ( 
$\frac{\rho_{l}^{2} g L_{c}^{3}}{\mu^{2}}$, indicating the relative importance of buoyancy and viscous forces) is at an order of $10^{4}-10^{5}$ in the bubble size range of our interest, so the effects of viscous forces on the bubble size are assumed to be negligible in terms of comparing the rising and dragging forces. Therefore the liquid flow field distant from the bubble surface is assumed inviscid. However, this does not mean the liquid viscous effects on bubble size close to the bubble surface can be neglected due to the existence of a boundary layer. The surface tension of the liquid may also play an important role in determining the size of the bubbles, which makes it very challenging to give analytical models and solutions. Hence, this paper resorts to semi-empirical equations incorporating dimensionless groups such as Morton and Eo numbers that reflect the relative importance of surface tension, viscous forces, and other influencing factors.

For the gas, it was assumed to be ideal and the flow was assumed to be incompressible. Since the gas injection rate was constant, it was assumed that the bubble size was proportional to the flow rate with no allowance for a change in bubble cross-section during ascension. Additionally, the motion of the bubble was not affected by the presence of another bubble immediately above it. During motion, the gas-liquid interface was acted on by a pressure difference between the gas and liquid and by surface tension forces, which are constant and uniform. Also, the operation was assumed to be isothermal in order to reduce the modeling complexity arising from the interactions of heat and mass transfer. The isothermal conditions were controlled in the experiments when the volumetric ratio of gas bubbles to the liquid phase is smaller than 0.0053. In the experiments, the liquid phase temperature fluctuations were observed within only $0.2{ }^{\circ} \mathrm{C}$, so the isothermal assumption was validated.

\subsection{Mass Transfer Across Interfaces in Two-phase Gas-liquid Flows}

Mass transfer between a droplet and a fluid has been extensively studied in the past. Theoretical models for mass transfer rate predictions from rising bubbles have been developed. Some of these models have been used for non-Newtonian fluids [27, 28], whereas others for different Reynolds numbers as demonstrated in [29-36]. Analogous to heat transfer, mass transfer occurs due to diffusion and convection.

Different types of diffusion have been reported in the past [37]. Diffusion takes place when the primary driving mechanism is a concentration gradient.Convection mass transfer refers to the transfer of mass between a surface and a moving medium as a result of both mass diffusion and bulk fluid motion [37]. Similar to heat transfer, mass transfer is influenced by surface geometry, flow velocity, flow regime, fluid properties and composition. Due to the processes that arise as a result of fluid motion and its properties, mass transfer is determined based on experimental data. Therefore, 
the relative magnitude of molecular momentum and mass diffusion in the velocity and concentration boundary layers are expressed by the dimensionless Schmidt number defined as follows [37]:

$$
\text { Sc }=\frac{v}{\alpha}
$$

In heat transfer analysis, it is convenient to express the heat transfer coefficient in terms of the dimensionless Nusselt number. Likewise, in mass transfer, the mass transfer coefficient is expressed in terms of the dimensionless Sherwood number [37]:

$$
\mathrm{Sh}=\frac{h_{\text {mass }} L_{c}}{\alpha}
$$

where $h_{\text {mass }}$ is the mass transfer coefficient in units of $\mathrm{m} / \mathrm{s}$ primarily indicating the convective contribution, and $\alpha$ is the molecular diffusivity in units of $\mathrm{m}^{2} / \mathrm{s}$ indicating the diffusive contribution, obtained from [37].

For an internal flow and using bulk motion properties, the above relation can be rewritten as:

$$
\left.\frac{d\left[\left(w_{A}-w_{A, s}\right) /\left(w_{A, \infty}-w_{A, s}\right)\right]}{d\left(y / L_{c}\right)}\right|_{y=0}=\frac{h_{\text {mass }} L_{c}}{\alpha}=S h
$$

Another method to determine the Sherwood number is given in terms of the Reynolds and Schmidt numbers. The expression is given as follows [38]:

$$
\mathrm{Sh}=\mathrm{cRe}^{1 / 2} \mathrm{Sc}^{1 / 3}
$$

where $c \sim 0.6$ is a constant. This expression is used when the bubble can be assumed to be rigid and non-deforming.

\subsection{Non-dimensional Correlations}

Past studies have shown that the Reynolds (Re), Eotvos (Eo) and Morton (Mo) numbers are useful for describing a rising bubble motion because the shape and terminal velocity of the bubble are influenced by these three dimensionless parameters [26]. In order to better understand how the three numbers describe the bubble's motion, it is necessary to determine their values. The Reynolds number is the ratio of inertial forces to viscous forces in the liquid:

$$
\operatorname{Re}=\frac{V L_{c}}{v}=\frac{\rho V L_{c}}{\mu}
$$

where $V$ is the bubble's velocity in $\mathrm{m} / \mathrm{s}, L_{c}$ is the diameter of the bubble in units of $\mathrm{m}, \mu$ is the dynamic viscosity of the liquid in units of Pa.s, $\rho$ is the density of the liquid in units of $\mathrm{kg} / \mathrm{m}^{3}$ and $v=\frac{\mu}{\rho}$ is the kinematic viscosity of the liquid in units of $\mathrm{m}^{2} / \mathrm{s}$. The Eotvos number characterizes the 
size and shape of the bubble and it is proportional to the buoyancy force divided by the surface tension force:

$$
\text { Eo }=\frac{\Delta \rho g L_{c}}{\sigma}
$$

where $\Delta \rho$ is the density change of the two phases and $\sigma$ is the surface tension in units of $\mathrm{N} / \mathrm{m}$. The Morton number is used together with the Eo number in order to characterize the bubble's shape:

$$
\text { Mo }=\frac{g \mu^{4} \Delta \rho}{\rho_{l}^{2} \sigma^{3}}
$$

When a drop deforms, its surface area increases with respect to a spherical drop of the same volume. Mass transfer between a droplet and a liquid is proportional to the surface area of the droplet [35].

In order to develop the correlation, Sh was first calculated based on Eq. (8), where Re is given by Eq. (9) and Sc is calculated using Eq. (5). Since the applicable scope of $\alpha$ in literature is limited, this paper aims to generalize it and extend it to bubble shapes and distance traveled by the bubble. As a result, the novel correlation can give information on mass transfer when $\alpha$ and $h_{\text {mass }}$ are unknown based on shape/size of bubbles and the bubbles rising distance.

The Buckingham PI theorem was employed to determine whether Sh can be correlated with the aforementioned parameters. The non-dimensional correlation will have the following form [39]:

$$
\mathrm{y}=\mathrm{m}_{0} \mathrm{t}_{1}^{\mathrm{m}_{1}} \mathrm{t}_{2}^{\mathrm{m}_{2} \ldots \mathrm{t}_{\mathrm{n}}} \mathrm{m}_{\mathrm{n}}
$$

where values of $t$ are the various independent parameters influencing the mass transfer and $m$ are the coefficients. Mass transfer in bubbles is dependent on different variables such as the surface tension, viscosity, velocity of the rising bubble, size and shape of the bubble, distance it travels and the temperature of the surrounding liquid. Table 3 lists the key parameters needed for the analysis of mass transfer across the interface, their units and dimensions in terms of mass (M), length (L), time (T) and temperature $(\mathrm{K})$.

An equation expressing the mass transfer coefficient as a function of the mass transfer parameters can be written as follows:

$$
h_{\text {mass }}=f(\mu, \sigma, V, D, d, \alpha, g, H, \rho)
$$

Eq. (13) can also be written as:

$$
h_{\text {mass }}=\frac{\mu}{\rho D}\left(\frac{d}{D}\right)^{e}\left(\frac{\rho D V}{\mu}\right)^{f}\left(\frac{\rho \alpha}{\mu}\right)^{h}\left(\frac{\rho D \sigma}{\mu^{2}}\right)^{i}\left(\frac{\rho^{2} D^{3} g}{\mu^{2}}\right)^{j}\left(\frac{H}{D}\right)^{l}
$$

After investigating Eq. (14), five dimensionless IIterms were determined, which are expressed in Table 4. Thus, the following equation was obtained based on the dimensional analysis:

$$
\mathrm{Sh}=\operatorname{Re}^{m_{1}} \operatorname{Eo}^{m_{2}}\left(\frac{d}{D}\right)^{m_{3}}\left(\frac{H}{D}\right)^{m_{4}}
$$


where $\mathrm{m}_{1}=\frac{\mathrm{f}}{1-\mathrm{h}}, \mathrm{m}_{2}=\frac{\mathrm{j}-\mathrm{i}}{1-\mathrm{h}}, \mathrm{m}_{3}=\frac{\mathrm{e}}{1-\mathrm{h}}, \mathrm{m}_{4}=\frac{1}{1-\mathrm{h}}$ are coefficients to be determined. In order to determine the coefficients in Eq. (15), experimental data were utilized. Diameters of gas bubbles were obtained using DynamicStudio using the steps described in the previous section.

\section{RESULTS AND DISCUSSION}

DynamicStudio software, an advanced edge detection algorithm to extract the bubble shape information, was used to acquire and process the images. The "shadow sizer processing” method was used to extract information such as size, position, shape and velocity of bubbles. Prior to image processing, a set of calibration images was acquired. This step was necessary because it enables the measurement of a scale factor. The scale factor was used to determine the conversion of pixel units into metric units. Figure 2 depicts the acquired image at a flow rate of $2 \mathrm{~L} / \mathrm{min}$. It can be seen that the gas bubbles are irregular in shape and their diameters are different. Due to their irregular shape, it is expected that more mass is transferred across the boundary compared to spherical bubbles. It is important to note that bubbles rise up in a spiral motion which enhances the mass transfer across the interface. After aquiring the images, they were processed using the Shadow Image module of DynamicStudio. In order to ensure that all gas bubbles were analyzed by the software, a grey level profile of the bubbles was determined.

The images were then processed in order to obtain useful information such as diameter, area, shape, position and velocity of the bubbles. The velocity information was extracted by combining a correlation algorithm and measuring displacement between two successive images via a dedicated particle-tracking algorithm. Figure 3 depicts the processed image with velocity vectors. These results have been used to calculate the Sherwood, Reynolds and Eotvos numbers in order to develop correlations that determine the amount of mass transfer across the interface of the gas bubble as it rises through the liquid. Diffusive mass transfer occurs across the interfacial boundaries of the gas bubbles.

Once the diameters of different size gas bubbles were obtained using DynamicStudio, it was possible to calculate the three dimensionless groups represented in Table 4. The dimensionless groups were calculated using MATLAB. Equations (8) through (10) were used to calculate the Reynolds number, Eotvos number and Sherwood number. Scatter plots were then generated using MATLAB. A relationship between Sh and Re, Sh and Eo and Sh and H/D can be observed in Figures 4, 5 and 7, respectively. No relationship observed between Sh and $d / D$ (see Figure 6) therefore it is expected that the corresponding term in Eq. (15) does not influence the correlation. From Figure 7 it can be deduced that Sh number increases as the diameter of the bubbles increases. Although a negative exponent would be expected due to the decreasing function represented in Figure 7, the correlation returned a 
positive exponent for $H / D$. This is due to the antecedent terms being a function of the diameter of the bubbles and the distance traveled, therefore the terms of the correlation are not independent of each other. Figure 8 shows the plot of $\mathrm{Sh} / \mathrm{Re}^{0.6267} \mathrm{Eo}^{0.4659}$ in order to demonstrate the dependency of the developed correlation on the diameter and distance traveled by the bubbles. MATLAB Optimization toolbox was employed to optimize exponents $\mathrm{m}_{1}, \mathrm{~m}_{2}, \mathrm{~m}_{3}$, and $\mathrm{m}_{4}$. Multifunction Genetic Algorithm was utilized and exponents were obtained as given in Eq. (16).

$\mathrm{Sh}=\operatorname{Re}^{0.6267} \mathrm{Eo}^{0.4659}\left(\frac{d}{D}\right)^{0}\left(\frac{H}{D}\right)^{0.5754}$

As expected, after optimization $\mathrm{m}_{3}=0$ therefore the term $d / D$ does not influence the correlation. The developed model was then tested and the results are given in Figures 9 and 10. This experimental data and correlation provide useful information for better understanding of diffusive mass transfer through bubbles, particularly for applications related to hydrogen production.

\section{CONCLUSIONS}

Water vapour entrainment in gas-liquid two-phase flow is a common mass and heat transport phenomenon existing in most water splitting processes for hydrogen production. This paper has examined the relations between the flow dynamics and mass transfer kinetics of multiphase flows involving inert gas bubbles and the amount of water vapour entrained by the gas. Experimental data were obtained for the change of bubble humidity and velocity to simulate the bubbles rising in a quiescent liquid. A new correlation was developed to relate the bubble dynamics and water vapour generation rate with Reynolds and Sherwood numbers particularly involving the amount of mass transferred across the interfacial boundary. The experimental data and model presented in this paper can provide a better understanding of the gas bubble dynamics and water vapour generation kinetics related to hydrogen production.

\section{ACKNOWLEDGEMENTS}

Financial support from the Ontario Research Excellence Fund and Atomic Energy of Canada Limited (AECL) is gratefully acknowledged.

\section{NOMENCLATURE}

d

D

$\alpha$

Eo

$\mathrm{h}_{\text {mass }}$
Minor diameter of bubble (m)

Major diameter of bubble (m)

Diffusivity $\left(\mathrm{m}^{2} / \mathrm{s}\right)$

Eotvos number

Mass transfer coefficient (m/s) 


$\begin{array}{ll}\mathrm{H} & \text { Water level (m) } \\ \mathrm{j}_{\mathrm{A}, \mathrm{i}} & \text { Diffusion flux (mol/m² }) \\ \mathrm{L} & \text { Length (m) } \\ \mathrm{m}_{\mathrm{n}} & \text { Coefficient, } \mathrm{n}=1,2,3,4 \\ \mathrm{M} & \text { Mass (kg) } \\ \mathrm{Mo} & \text { Morton number } \\ \mathrm{Re} & \text { Reynolds number } \\ \mathrm{Sc} & \text { Schmidt number } \\ \mathrm{Sh} & \text { Sherwood number } \\ \mathrm{V} & \text { Velocity (m/s) } \\ \mathrm{w} & \text { Mass mole fraction }\end{array}$

\section{Greek Symbols}

$\begin{array}{cl}\mu & \text { Dynamic viscosity of a fluid }\left(\frac{\mathrm{kg}}{\mathrm{m} \cdot \mathrm{s}}\right) \\ v & \text { Kinematic viscosity }\left(\mathrm{m}^{2} / \mathrm{s}\right) \\ \rho & \text { Density }\left(\mathrm{kg} / \mathrm{m}^{3}\right) \\ \sigma & \text { Surface tension }(\mathrm{N} / \mathrm{m})\end{array}$

\section{Subscripts}

$\begin{array}{ll}\text { A } & \text { Species A } \\ \text { B } & \text { Species B } \\ \text { C } & \text { Characteristic } \\ \text { l } & \text { Surrounding liquid } \\ \text { S } & \text { Surface }\end{array}$

\section{REFERENCES}

[1] I. Dincer, Green methods for hydrogen production. International Journal of Hydrogen Energy 37 (2012) 1954-1971.

[2] M.A. Rosen, Advances in hydrogen production by thermochemical water decomposition: A review. Energy 35 (2010) 1068-1076.

[3] G.F. Naterer, S. Suppiah, L. Stolberg, M. Lewis, M. Ferrandon, Z. Wang, et al. Clean hydrogen production with the $\mathrm{Cu}-\mathrm{Cl}$ cycle - Progress of international consortium, I: Experimental unit operations. International Journal of Hydrogen Energy 35 (2011) 15472-15485.

[4] G. Naterer, S. Suppiah, M. Lewis, K. Gabriel, I. Dincer, M.A. Rosen, et al. Recent Canadian advances in nuclear-based hydrogen production and the thermochemical $\mathrm{Cu}-\mathrm{Cl}$ cycle. International Journal of Hydrogen Energy 34 (2009) 2901-2917. 
[5] J. O’M. Bockris, B. Bandapani, D. Cocke, J. Ghoroghchian, On the splitting of water. International Journal of Hydrogen Energy 10 (1985) 179-201.

[6] Z. Wang, R.R. Roberts, G.F. Naterer, K.S. Gabriel, Comparison of thermochemical, electrolytic, photoelectrolytic and photochemical solar-to-hydrogen production technologies. International Journal of Hydrogen Energy 37 (2012) 16287-16301.

[7] M.D. Mat, K. Aldasb, Application of a two-phase flow model for natural convection in an electrochemical cell. International Journal of Hydrogen Energy 30 (2005) 411-420.

[8] P. Boissonneau, P. Byrne, An experimental investigation of bubble-induced free convection in a small electrochemical cell. Journal of Applied Electrochemistry 30 (2000) 767-775.

[9] A.A. Dahlkild, Modeling the two-phase flow and current distribution along a vertical gas-evolving electrode. Journal of Fluid Mechanics 428 (2001) 249-272.

[10] B.A. Ali, S. Pushpavanam, Analysis of liquid circulation and mixing in a partitioned electrolytic tank. International Journal of Multiphase Flow 37 (2011) 1191 - 1200.

[11] J.M. Norbeck, J.W. Heffel, T. D. Durbin, B. Tabbara, J.M. Bowden, M.C. Montani, Hydrogen Fuel for Surface Transportation. Society of Automotive Engineers Inc. Warrandale, PA, 1996.

[12] R. Clift, J.R. Grace, M.E. Weber, Bubbles, Drops and Particles, Academic Press, New York, 1978. (republished by Dover in 2005).

[13] P.C. Duineveld, The rise velocity and shape of bubbles in pure water at high Reynolds number. Journal of Fluid Mechanics 292 (1995) 325-332.

[14] K. Ellingsen, F. Risso, On the rise of an ellipsoidal bubble in water: oscillatory paths and liquid induced velocity. Journal of Fluid Mechanics 440 (2001) 235-268.

[15] J. Magnaudet, I. Eames, The motion of high-Reynolds-number bubbles in inhomogeneous flows. Annual Review of Fluid Mechanics 32 (2000) 659-708.

[16] N.M.S. Hassan, M.M.K. Khan, M.G. Rasul, A modelling and experimental study of the bubble trajectory in a non-Newtonian crystal suspension. Fluid Dynamics Research 42 (2010) 065502.

[17] A.A. Kulkarni, J.B. Joshi, Bubble formation and bubble rise velocity in gas-liquid systems: A review. Industrial and Engineering Chemistry Research 44 (2005) 5873-5931.

[18] T. Miyahara, T. Takahashi, Coalescence phenomena at the moment of bubble formation at adjacent holes. Chemical Engineering Research and Design 64 (1986) 320-323.

[19] M. Ohta, D. Kikuchi, Y. Yoshida, M. Sussman, Robust numerical analysis of the dynamic bubble formation process in a viscous liquid. International Journal of Multiphase Flow 37 (2011) 1059-1071.

[20] V.V. Buwa, D.S. Deo, V.V. Ranade, Eulerian-Lagrangian simulations of unsteady gas-liquid flows in a bubble column. International Journal of Multiphase Flow 32 (2006) 864-885. 
[21] B. Yang, Numerical studies of single gas and vapor bubble flows. PhD thesis, The Johns Hopkins University, Baltimore, MD, 2006.

[22] N.M. Aybers, A. Tapucu, The motion of gas bubbles rising through stagnant liquid. Heat and Mass Transfer 2 (1969) 118-128.

[23] O. Jianu, M.A. Rosen, G.F. Naterer, Z. Wang, Mass Transfer and Bubble Flow Dynamics in Aqueous Solutions for Hydrogen Production Cycles. Proc. $11^{\text {th }}$ International Conference on Sustainable Energy Technologies. Vancouver, BC 2012, pp. 2244-2255.

[24] S.J. Kline, F.A. McClintock Describing Uncertainties in Single-Sample Experiments. Mechanical Engineering 75 (1953) 3-8.

[25] R.J. Moffat, Contributions to the Theory of Single-Sample Uncertainty Analysis, Transactions of the ASME, 104 (1982) 250-258.

[26] R.J. Moffat, Using Uncertainty Analysis in the Planning of an Experiment, Journal of Fluids Engineering, 107 (1985) 173-178.

[27] Y. Kawase, Ulbrecht, J.J. Drag and mass transfer in non-Newtonian flows through multiparticle systems at low Reynolds numbers, Chemical Engineering Science 36 (1981) 11931202.

[28] Y. Kawase, M. Moo-Young, Approximate solutions for power law fluid flow past a particle at low Reynolds numbers, Journal of Non-Newtonian Fluid Mechanics 21 (1986) 167-177.

[28] A.B. Jarzebski, J.J. Malinowski, Transient mass and heat transfer from drops or bubbles in slow non-Newtonian flows, Chemical Engineering Science 41 (1986) 2575-2578.

[30] Z.G. Feng, E.E. Michaelides, Mass and heat transfer from fluid spheres at low Reynolds numbers, Powder Technology 112 (2000) 63-69.

[31] M. Favelukis, S. Raphael, Mass transfer between a slender bubble and a viscous liquid in axisymmetric extensional flow, Chemical Engineering Science 51 (1996) 1169-1172.

[32] E. Ruckenstein, Prediction of rates of heat or mass transfer in complex situations by interpolating between simpler limiting cases, Chemical Engineering Science 37 (1982) 15051511.

[33] Y. Nakano, C. Tien, Approximate solutions of viscous incompressible flow around fluid spheres at intermediate Reynolds numbers, Canadian Journal of Chemical Engineering 45 (1967) 135-140.

[34] N. Kishore, R.P. Chhabra, V. Eswaran, Bubble swarms in power-law liquids at moderate Reynolds numbers: Drag and mass transfer. Chemical Engineering Research and Design 86 (2008) 39-53. 
[35] M. Martin, F. J. Montes, M.A. Galan, Approximate theoretical solution for the Sherwood number of oscillating bubbles at different Reynolds numbers. Chemical Engineering and Processing 49 (2010) 245-254.

[36] M.H.L. Baird, A.E. Hamielec, Forced convection transfer around sphere at intermediate Reynolds numbers, Can. J. Chem. Eng. 40 (1962) 119-121.

[37] Y.A. Cengel, Heat and Mass Transfer: A practical Approach, third ed. McGraw Hill 2007, pp.774-783.

[38] J.M.T. Vasconcelos, S.P. Orvalho, S.S. Alves, Gas-Liquid Mass Transfer to Single Bubbles: Effect of Surface Contamination. American Institute of Chemical Engineers Journal 48 (2002) $1145-1154$.

[39] X. Wang, G.F. Naterer, E. Bibeau, Convective Droplet Impact and Heat Transfer from a NACA Airfoil. Journal of Thermophysics and Heat Transfer 21 (2007) 536-542. 


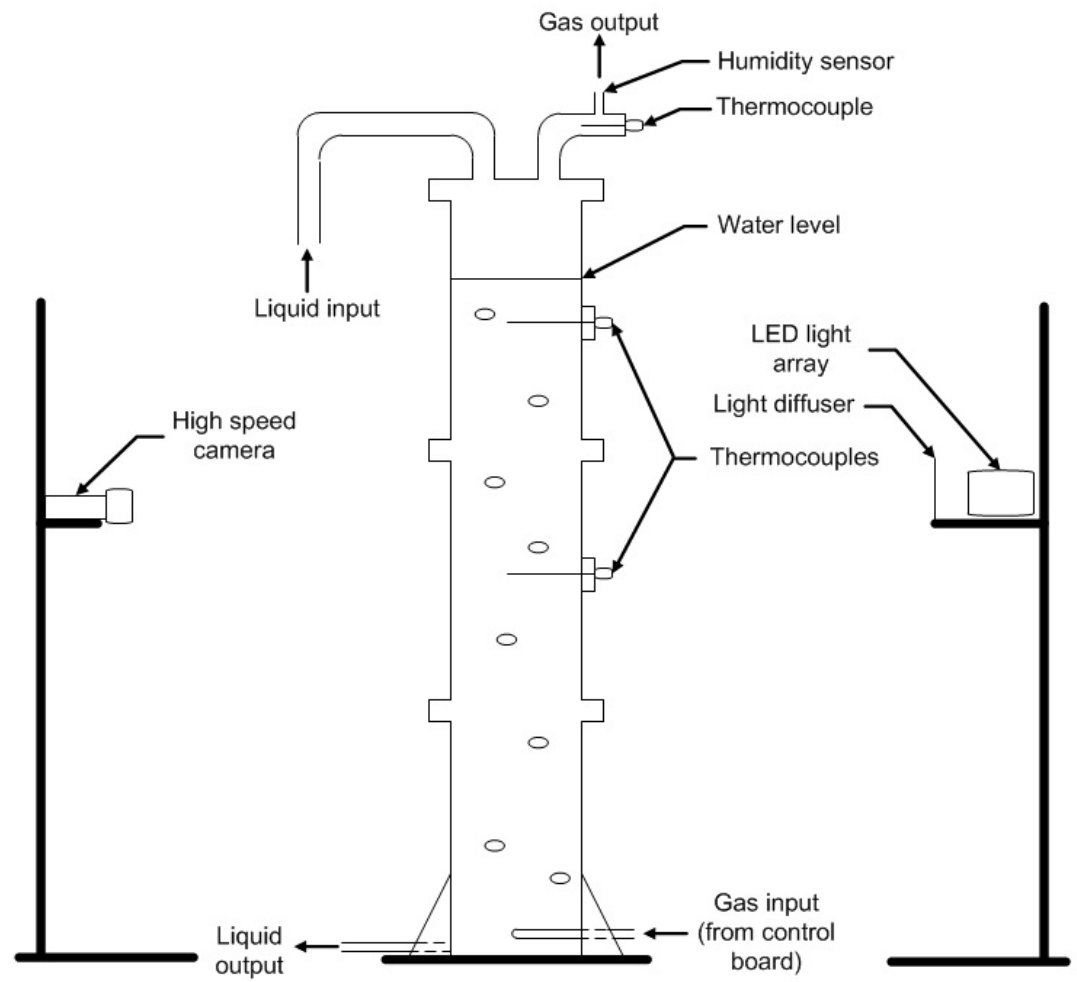

Fig. 1. Schematic of experimental setup

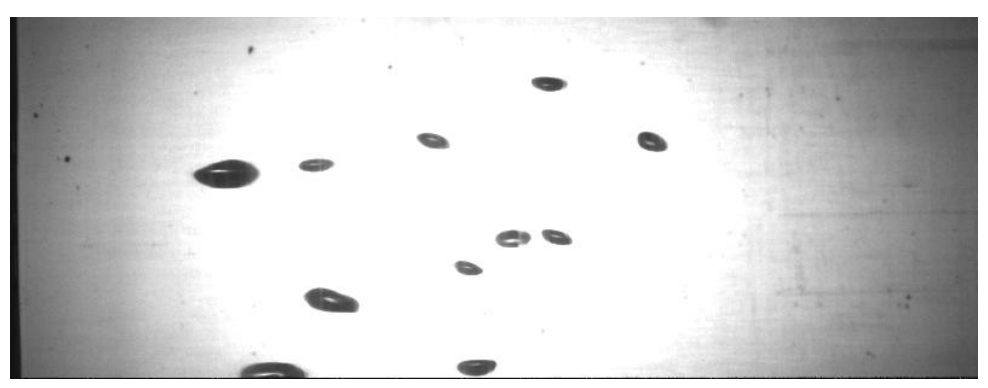

Fig. 2. Aquired image for $2 \mathrm{~L} / \mathrm{min}$

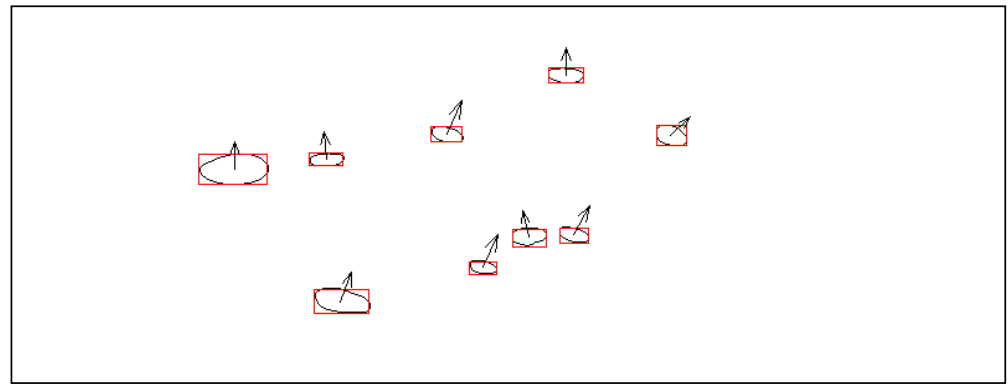

Fig. 3. Processed image for $2 \mathrm{~L} / \mathrm{min}$ 


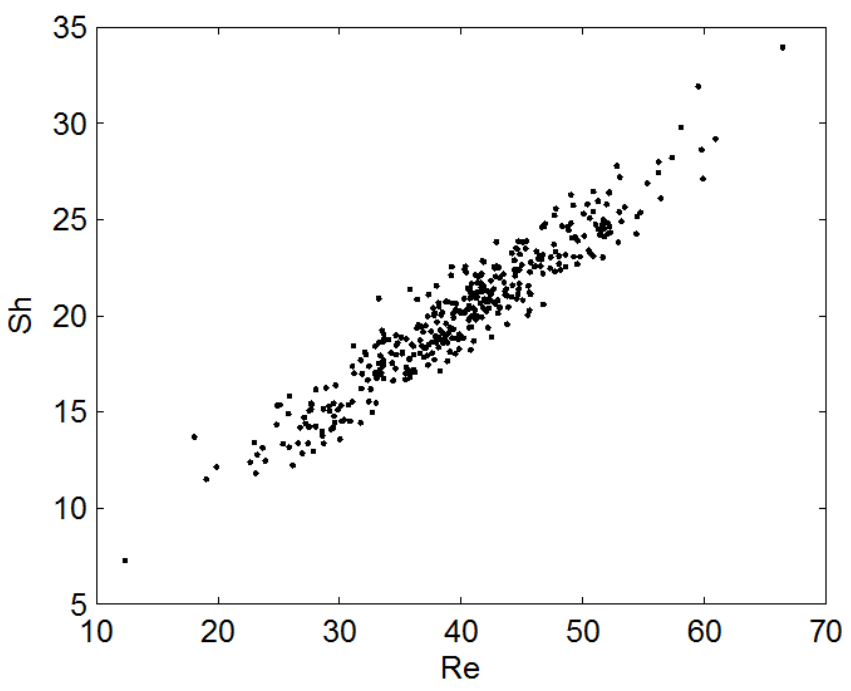

Fig. 4. Scatter plot for Re versus Sh

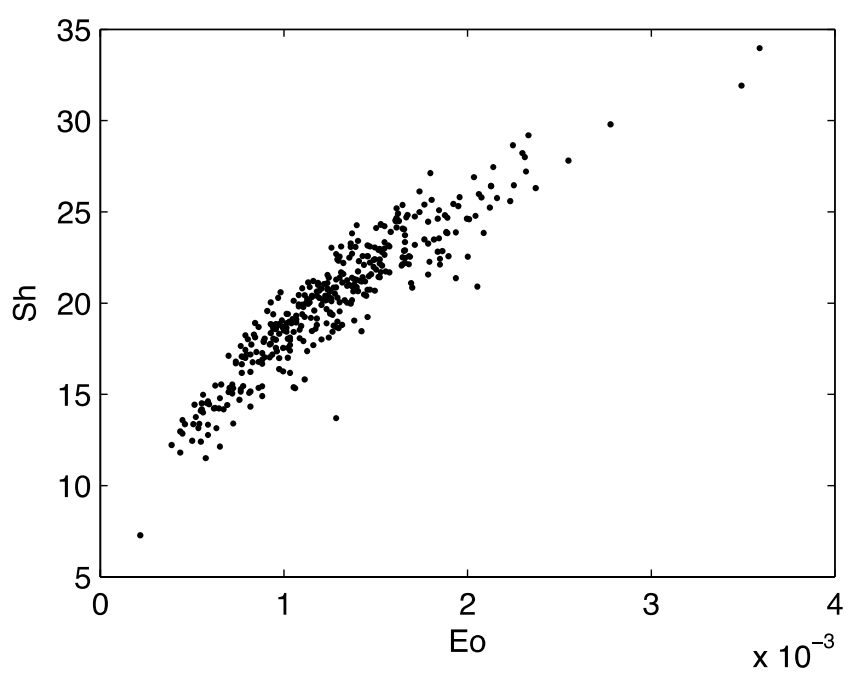

Fig. 5. Scatter plot for Eo versus Sh

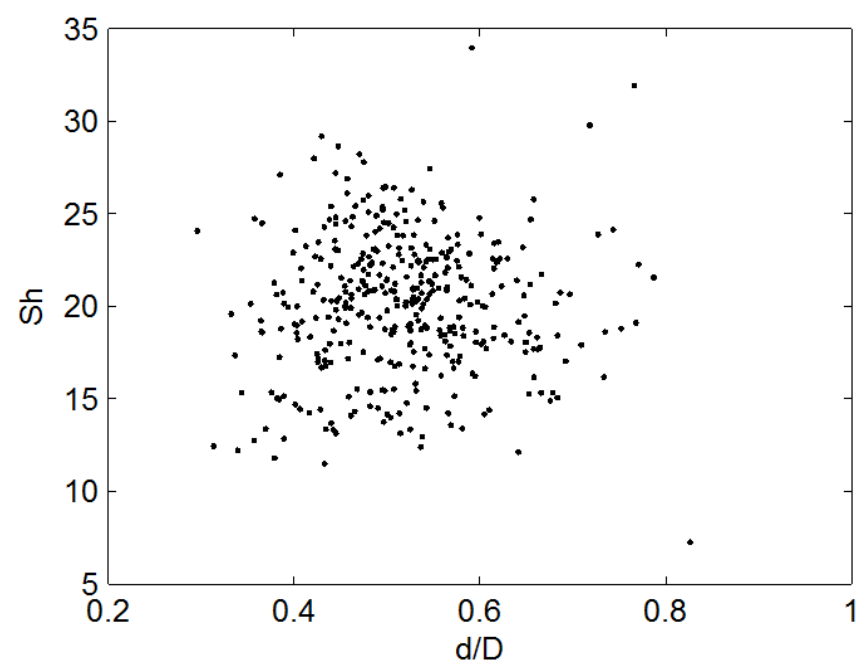

Fig. 6. Scatter plot for d/D versus Sh 


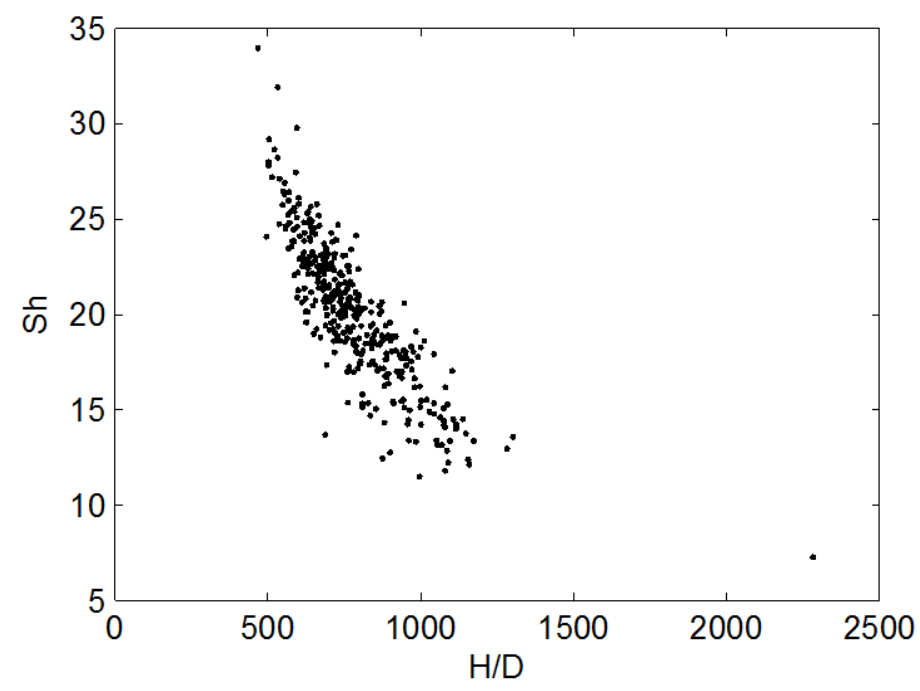

Fig. 7. Scatter plot for H/D versus Sh

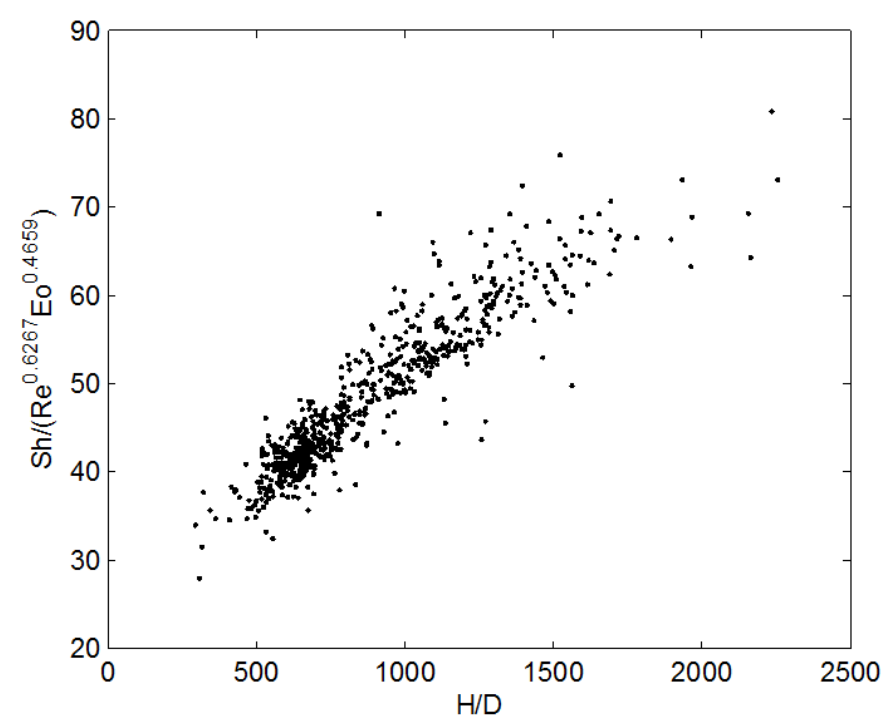

Fig. 8. Scatter plot for H/D versus Sh/Re $\mathrm{R}^{0.6267} \mathrm{Eo}^{0.4659}$

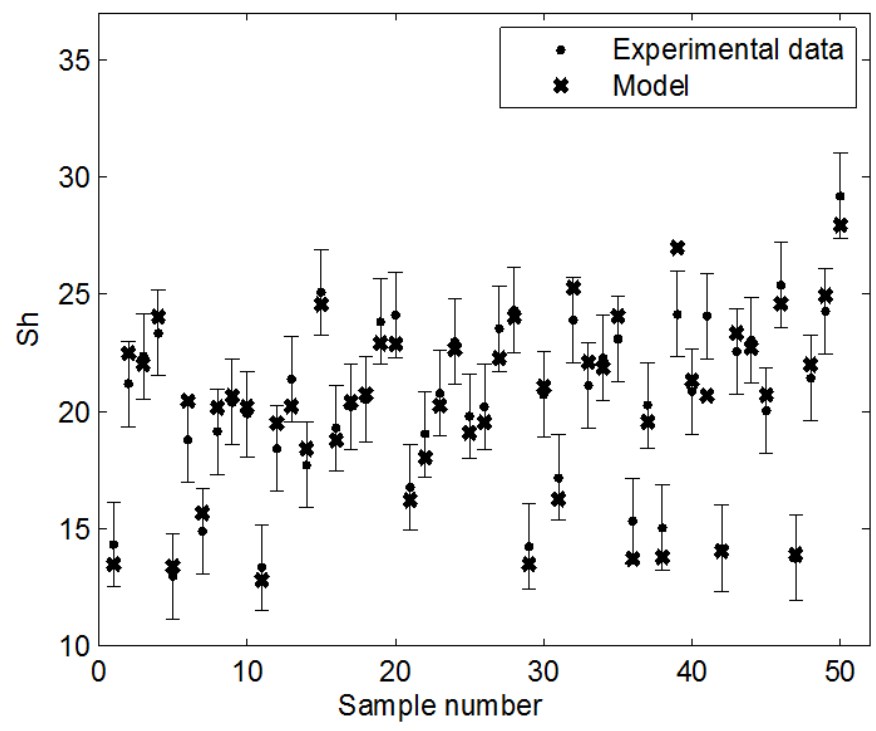

Fig. 9. Actual and modeled Sherwood number at $0.25 \mathrm{~L} / \mathrm{min}$ 


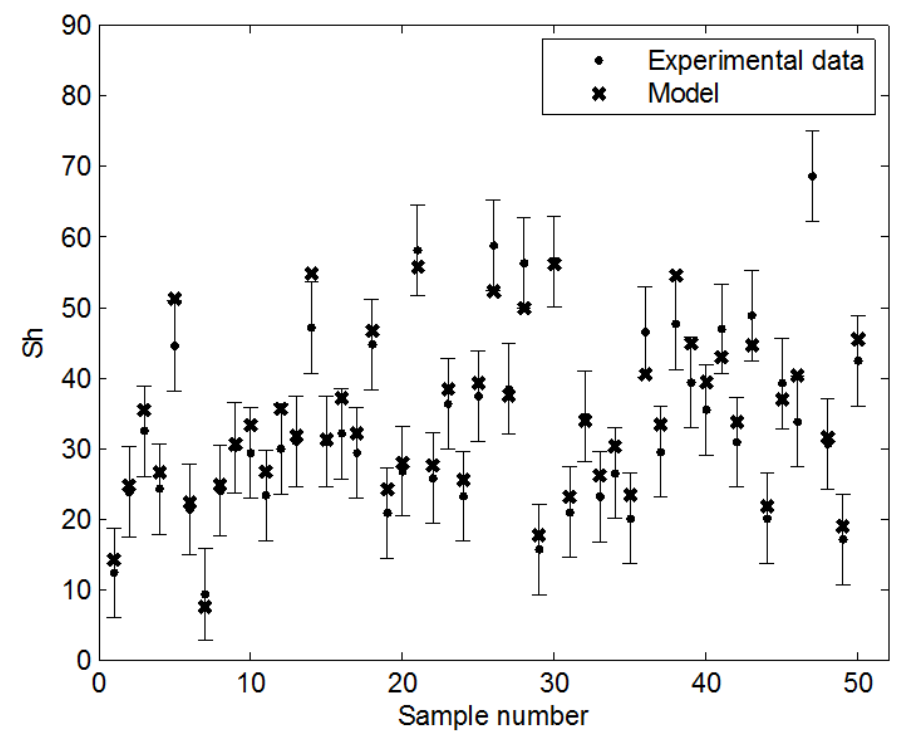

Fig. 10. Actual and modeled Sherwood number at $3 \mathrm{~L} / \mathrm{min}$

Table 1 - Results with DynamicStudio for $2 \mathrm{~L} / \mathrm{min}$

\begin{tabular}{|c|c|c|c|c|c|c|c|c|}
\hline $\begin{array}{l}\text { Centroid } X^{*} \\
(\mathrm{~mm})\end{array}$ & $\begin{array}{c}\text { Centroid } Y^{*} \\
(\mathrm{~mm})\end{array}$ & $\begin{array}{c}\text { Area } \\
\left(\mathrm{mm}^{2}\right)\end{array}$ & $\begin{array}{c}\text { Perimeter } \\
(\mathrm{mm})\end{array}$ & \begin{tabular}{|c|}
$\begin{array}{c}\text { Equivalent } \\
\text { Diameter } \\
(\mathrm{mm})\end{array}$ \\
\end{tabular} & $\begin{array}{l}\text { Major } \\
\text { Axis** } \\
(\mathrm{mm}) \\
\end{array}$ & $\begin{array}{l}\text { Minor } \\
\text { Axis*** } \\
(\mathrm{mm})\end{array}$ & \begin{tabular}{|c|} 
X-direction \\
velocity \\
$(\mathrm{m} / \mathrm{s})$
\end{tabular} & $\begin{array}{c}\begin{array}{c}\text { Y-direction } \\
\text { velocity } \\
(\mathrm{m} / \mathrm{s})\end{array} \\
\end{array}$ \\
\hline 27.18 & 19.87 & 166.70 & 18.30 & 5.69 & 4.28 & 1.90 & 0.00 & 0.30 \\
\hline 38.49 & 18.68 & 38.59 & 9.30 & 2.74 & 2.30 & 0.82 & -0.03 & 0.31 \\
\hline 40.19 & 35.89 & 110.11 & 15.10 & 4.62 & 3.66 & 1.49 & 0.12 & 0.32 \\
\hline 53.20 & 15.62 & 40.87 & 8.69 & 2.82 & 2.10 & 0.96 & 0.16 & 0.37 \\
\hline 57.60 & 31.77 & 31.26 & 7.63 & 2.46 & 1.80 & 0.86 & 0.16 & 0.36 \\
\hline 63.25 & 27.98 & 50.33 & 9.76 & 3.13 & 2.22 & 1.11 & -0.06 & 0.28 \\
\hline 67.83 & 8.42 & 46.21 & 9.61 & 3.00 & 2.35 & 0.97 & 0.01 & 0.30 \\
\hline 68.77 & 27.81 & 36.76 & 8.24 & 2.67 & 1.97 & 0.92 & 0.18 & 0.32 \\
\hline 80.72 & 15.78 & 53.23 & 9.15 & 3.21 & 2.06 & 1.28 & 0.21 & 0.21 \\
\hline
\end{tabular}

Table 2 - Experimental uncertainties

\begin{tabular}{|l|c|}
\hline Source of Error & Deviation \\
\hline Calibration & 0.019 \\
\hline Residual & 0.01 \\
\hline Resolution & 0.011 \\
\hline Vessel distortion & 0.005 \\
\hline Analysis & 0.009 \\
\hline Total & $\mathbf{0 . 0 5 4}$ \\
\hline
\end{tabular}


Table 3 - Various parameters influencing mass transfer

\begin{tabular}{|c|c|c|c|}
\hline $\begin{array}{l}\text { Factors Affecting } \\
\text { Mass Transfer }\end{array}$ & Units & MLT & Coefficient \\
\hline Dynamic viscosity & $\frac{k g}{s \cdot m}$ & $M T^{-1} L^{-1}$ & $\mathbf{a}$ \\
\hline Surface tension & $\frac{k g}{s^{2}}$ & $M T^{-2}$ & $\mathbf{b}$ \\
\hline Bubble velocity & $\frac{m}{s}$ & $L T^{-1}$ & c \\
\hline Major diameter & $m$ & $L$ & $\mathbf{e}$ \\
\hline Minor diameter & $m$ & $L$ & f \\
\hline Diffusivity & $\frac{m^{2}}{s}$ & $L^{2} T^{-1}$ & h \\
\hline Gravity & $\frac{m}{s^{2}}$ & $L T^{-2}$ & $\mathrm{i}$ \\
\hline Water level & $m$ & $L$ & j \\
\hline Density & $\frac{k g}{m^{3}}$ & $M L^{-3}$ & 1 \\
\hline $\begin{array}{l}\text { Mass transfer } \\
\text { coefficient }\end{array}$ & $\frac{m}{s}$ & $L T^{-1}$ & \\
\hline \multicolumn{4}{|c|}{$M=$ mass $; L=$ length $; T=$ time } \\
\hline
\end{tabular}

Table 4 - Correlations of $\Pi$ Terms

\begin{tabular}{|c|c|}
\hline PI Terms & Description \\
\hline$\frac{\Pi_{1}}{\Pi_{4}}=\frac{h_{\text {mass }} \mathrm{D}}{\mathrm{D}_{\mathrm{AB}}}$ & Sherwood number \\
\hline$\Pi_{2}=\frac{\mathrm{d}}{\mathrm{D}}$ & Aspect ratio \\
\hline$\Pi_{3}=\frac{\rho \mathrm{DV}}{\mu}$ & Reynolds number \\
\hline$\Pi_{6}=\frac{\rho \mathrm{D}^{2} \mathrm{~g}}{\sigma}$ & Eotvos number \\
\hline$\Pi_{5}$ & \\
\hline$\Pi_{7}=\frac{\mathrm{H}}{\mathrm{D}}$ & \\
\hline
\end{tabular}

